CA7-02:

\section{The Extent and Impact of Consistent Primary Care}

Thomas Flottemesch ${ }^{1}$; Louise Anderson ${ }^{1}$; Leif Solberg ${ }^{1}$; Patricia Fontaine ${ }^{2}$; Stephen Asche ${ }^{1}$

${ }^{1}$ HealthPartners; ${ }^{2}$ University of Minnesota

Background/Aims: Clinical practice systems are an important component of a high functioning Patient-Centered Medical Home (PCMH). Here, the relationships between PCMH clinical practice systems and healthcare costs and utilization are examined using a retrospectively constructed longitudinal cohort receiving consistent primary care from the same medical group over a 5-year period. Methods: A 2005 survey of medical group directors measured clinical practice systems using the Physician Practice Connections ${ }^{\circledR}$ Research Survey ${ }^{\mathrm{TM}}$ (PPC $\left.{ }^{\circledR}-\mathrm{RS}\right)$. Medical group PPC ${ }^{\circledR}$-RS scores were compared to the annual medical costs and utilization of a cohort of 58,391 persons attributed across 22 medical groups over a 5-year period (20052009). Four outcomes adjusted to 2005 dollars were considered: total annualized cost, total annualized outpatient cost, total annualized inpatient cost, and annualized emergency department visits (ED). Multivariate, multilevel regressions adjusting for patient demographics, health status and autoregressive errors estimated the relationship between PPC $($-RS and study outcomes. Cohort-wide associations with PPC $®$-RS scores as well as specific effects within three sub-cohorts (medication count: 0-2[N=29,657], $2-6[\mathrm{~N}=19,505], 7+[\mathrm{N}=9,229])$ were considered. Results: The only consistent, cohort-wide relationship was with ED use. A $10 \%$ (71.13 to 78.24) increase in PPC $®$-RS scores was associated with 3.9 (medication count: 0-2), 6 (medication count: 3-6), and 11.6 (medication count: 7+) fewer ED visits/1000 in 2005; and 5.1, 7.6, and 13.6 fewer visits/1000 in 2009. The

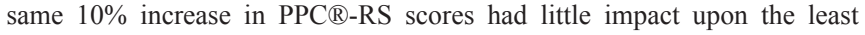
complex (medication count: $0-2)$ with no significant change in total ( $-\$ 22 /$ person in 2005; \$184/person in 2009), outpatient( $-\$ 11$ person in 2005; \$42/ person in 2009), and inpatient (\$26/person in 2005; \$29/person in 2009) costs. For medically complex patients (medication count: $7+$ ), the story was different. The $10 \%$ increase in PPS-RS scores was associated with significantly decreased total (\$446/person in 2005; \$184/person in 2009) and outpatient (\$241/person in 2005; \$54/person in 2009) costs, but no significant change in inpatient costs (\$43/person in 2005; $\$ 46 /$ person in 2009). Discussion: Improved PCMH clinical practice systems appear associated with a sustained reduced reliance upon emergency care, and their association with costs appears limited to the most medically complex who would most benefit from improved management of complex disease.

Keywords: Primary Care; Medical Home; Health Services Research doi:10.3121/cmr.2012.1100.ca7-02

CA7-03:

Adolescent and Adult Immunizations: Health Impact and CostEffectiveness

Louise Anderson ${ }^{1}$; Michael Maciosek ${ }^{1}$; Thomas Flottemesch ${ }^{1}$; Nichol Edwards ${ }^{1}$; Leif Solberg ${ }^{1}$

\section{${ }^{1}$ HealthPartners}

Background/Aims: The scope of vaccines recommended for adolescents and adults by ACIP has grown in number of vaccines and populations covered. We estimated the relative health impact and cost-effectiveness of these vaccines to help guide quality improvement initiative decisions. Methods: Markov deterministic and micro-simulation models were used to estimate the health impact and CE ratios for birth cohorts of four million using a societal perspective. All models used methods consistent with the 'reference case' of the Panel of Cost-Effectiveness in Health and Medicine, producing results that are comparable and suitable for ranking. The influenza model recognized the 2010 ACIP recommendation for universal vaccination. The pneumococcal model recognized the recent decline in adult incidence after introduction of the PCV7 childhood vaccine. The meningococcal model included the 2010 ACIP recommendation for a booster five years after an initial vaccination at age 11 or 12 . The HPV model estimated the influence of vaccine on rates of cervical cancer with and without cancer screening. Parameter estimates were from published literature. Single- and multiplevariable sensitivity analyses were performed. Results: Health impacts, measured as QALYs saved during the lifetime of the cohort, were greatest for influenza vaccine $(283,300)$, followed by HPV vaccine with screening $(221,100)$, pneumococcal vaccine $(17,100)$ and meningococcal vaccine $(2,900)$. CE ratios ranged from $\$ 9,300$ for adult pneumococcal vaccination to over $\$ 640,000$ for adolescent meningococcal vaccination. $\mathrm{CE}$ of influenza vaccination for ages 18 and older was $\$ 6,100$, but the vaccine was most costeffective for ages 65 and older $(\$ 4,000)$ and least cost-effective for ages 18 to $49(\$ 260,500)$. HPV vaccine CE was $\$ 15,100$ without screening and $\$ 17,900$ with screening. Results from micro-simulation of the zoster vaccine will also be presented. Discussion: Priority among preventive health care services is important because of the limited time and resources of the health care delivery system and the need to focus on the most effective services. Our consistent methods allow for comparison of estimates across a number of services. Our updated models showed the greatest health impact from HPV vaccine in the presence of screening and influenza vaccination of adults ages 65 and older.

Keywords: Cost-Effectiveness; Immunization; Health Services Research doi:10.3121/cmr.2012.1100.ca7-03

CA7-04:

Costs of Care for Persons with Opioid Dependence in Two Integrated Health Systems

Frances Lynch ${ }^{1}$; Dennis McCarty²; Jennifer Mertens ${ }^{3}$; Nancy Perrin ${ }^{1}$; Carla Green $^{1}$; Sujaya Parthasarathy ${ }^{3}$; Bradley Anderson ${ }^{1}$; Monika Koch ${ }^{3}$

${ }^{1}$ Kaiser Permanente Northwest; ${ }^{2}$ Oregon Health \& Science University; ${ }^{3}$ Kaiser Permanente Northern California

Background/Aims: Opioid dependence is a growing public health concern and results in high costs to individuals, health care systems, and society. Recent legislation allowing expansion of buprenorphine for replacement therapy use in general medical care settings may increase access to care for opioid dependence, but little is known about its impact on services utilization and commercial health systems costs. In this retrospective cohort study, we examined how the introduction of buprenorphine affected the pattern of medical care and addiction medicine (AM) services provided to patients with opioid dependence and associated costs. Methods: Using electronic health records, we identified individuals with two or more diagnoses of opioid dependence per year from 2000 through 2008 in two large non-profit, integrated health systems (System A: N=4,425; System B: N=7122) and assessed health system utilization and costs. Results: In both health systems and across the study period, the number of opioid-dependent persons increased considerably and the use of buprenorphine for opioid dependence treatment increased steadily. In System A, those receiving buprenorphine plus AM counseling had significantly higher costs than those receiving methadone plus AM counseling $(Z=-9.22, \mathrm{p}<.001)$, and significantly lower costs than those with little or no AM counseling $(\mathrm{Z}=2.81, \mathrm{p}=.005)$. There were no differences in costs between those receiving buprenorphine plus AM counseling and those with $\mathrm{AM}$ counseling only $(\mathrm{Z}=1.30, \mathrm{p}=.192)$. The treatment group by period interaction $(? 2=9.66, \mathrm{df}=3, \mathrm{p}=.022)$ was significant, with costs decreasing over time in the buprenorphine plus AM counseling group and increasing over time in all other groups. In System B, costs were significantly lower for the buprenorphine plus AM counseling group than for the group with little or no AM counseling $(Z=-5.14, p<.001)$ and higher than for the group with AM counseling only $(\mathrm{Z}=5.56, \mathrm{p}=.001)$. The treatment group by period interaction was not significant $(? 2=1.23$, $\mathrm{df}=2, \mathrm{p}=.540$ ). Discussion: Buprenorphine treatment is emerging as a viable alternative to other AM treatment approaches for persons with opioid dependence. Results of this study provide further evidence that buprenorphine treatment can be provided at a similar cost to alternative strategies in private integrated health systems.

Keywords: Costs; Opioid Dependence; Health Services Research doi:10.3121/cmr.2012.1100.ca7-04 